\title{
The Gravel Packing Length Determination Method and Influencing Factors Analysis in Deepwater Horizontal Wells
}

\author{
Hui Huang, ${ }^{1}$ Min Wen, ${ }^{1}$ Xuesong Xing, ${ }^{1}$ Hao Qiu $\mathbb{D},{ }^{1}$ Zening Hou, ${ }^{1}$ and Shengtian Zhou $\mathbb{D}^{2}$ \\ ${ }^{1}$ CNOOC Research Institute co., Ltd., Beijing 100028, China \\ ${ }^{2}$ China University of Petroleum, Shandong, Qingdao 266580, China
}

Correspondence should be addressed to Hao Qiu; haoqiu216@163.com

Received 23 October 2021; Accepted 8 December 2021; Published 18 January 2022

Academic Editor: Jinjie Wang

Copyright (c) 2022 Hui Huang et al. This is an open access article distributed under the Creative Commons Attribution License, which permits unrestricted use, distribution, and reproduction in any medium, provided the original work is properly cited.

\begin{abstract}
Horizontal well gravel packing is the most commonly used sand control technology in offshore oil and gas fields. For extreme conditions such as deepwater, low fracture pressure formations, and long horizontal well bore length, targeted and costeffective measures are required. According to the friction models in different stages of gravel packing process of horizontal well, the corresponding friction is calculated and compared. According to the calculation, during the entire packing process, the washpipe/screen annular friction is the largest in $\beta$ wave packing stage, which reflects that higher packing pressure is required in this stage, and the formation fracture pressure is easily broken at this stage. According to the equilibrium flow velocity, the calculation method and flow chart of $\alpha$-wave sand bed height were put forward. The criterion and calculation method of packing length were designed. The influencing factors of viscosity, density and leakage rate of carrier fluid on $\alpha$ wave packing length were discussed. The quantitative analysis was carried out. The design and calculation method of $\alpha$ - $\beta$ wave packing length considering the successful completion of $\alpha$ wave packing and the successful completion of $\beta$ wave reverse packing was put forward. The corresponding software was compiled to discuss and calculate the quantitative analysis of the factors affecting the $\alpha-\beta$ wave packing length, such as the density of carrier fluid, gravel density and washpipe/screen ratio. For specific conditions, certain criteria and methods can be used to design and optimize horizontal well gravel packing length.
\end{abstract}

\section{Introduction}

With the increase of oil and natural gas demand and the progress of horizontal well drilling and completion technology, increasing the length of horizontal well to improve production has become increasingly popular, especially in the development of offshore oil and gas fields. In order to reduce the number of offshore platforms and costs, horizontal well technology has been used extensively. However, for offshore oil and gas fields, most of them are in extreme conditions, such as deepwater, ultra-deepwater, long or ultra-long horizontal wellbore section, and unconsolidated oil and gas reservoirs with low fracture pressure gradient. Most of these wells require sand control, and horizontal well gravel pack sand control is the most common completion method. Horizontal well gravel pack consists of two important stages, namely the $\alpha$ wave packing stage and $\beta$ wave packing stage [1]. The $\alpha$ wave packing stage is the forward packing process for the equilibrium dune at the bottom of the wellbore [2]. When $\alpha$ wave packing front reaches the toe of the horizontal wellbore or when the top of the sedimentary sand bed reaches the upper of the casing or wellbore, gravel particles in slurry cannot move forward, at this point the $\alpha$ wave packing stage stop. Gravel particles begin to reverse pack from the toe of the wellbore in the upper part of the already formed $\alpha$ wave sand bed to the heel of the horizontal section. This process is called the $\beta$-wave pack stage. The carrier fluid enters the washpipe/screen annulus through the deposited gravel and screen, enters the washpipe at the inlet of the wellbore toe, and returns to the surface.

Although the horizontal gravel pack has been widely recognized as a reliable sand control technology, there are significant risks associated with current horizontal gravel pack operations. For extreme reservoir conditions, if wellbore length is short, the expected production will not be achieved. However, if the horizontal wellbore length is long, the height 
of sand bed may be too high in the process of $\alpha$ wave packing stage, or the formation fracture pressure may be exceeded in $\beta$ wave packing stage. So the entire horizontal wellbore section cannot be fully packed and effective sand control effect cannot be achieved [3]. Especially for the unconsolidated sandstone reservoir, the long horizontal well, the deepwater and low fracture pressure formations. Premature sand bridge may occur before the gravel pack reaches the toe of the horizontal section, resulting in failure of the operation. This is mainly because the horizontal wellbore packing section is long, and sufficient pump rate is required to transport gravel to the toe of the wellbore, which results in higher pump pressure. The formation can be easily fractured due to low fracture gradient, resulting in the leakoff of carrier fluid to the formation during the packing process. If the pump rate is low, the carrying capacity of carrier fluid to carry gravel is insufficient. Both of these conditions can lead to premature bridging and pack failure, which can make the packing process very complicated and difficult to control. The gravel packing length is a particulary important for the target. If wellbore length design is too short to meet its expected production, if the wellbore length is too long to complete entire gravel packing. To date, there is no design and calculation method for gravel packing length in horizontal wells. Therefore, for specific conditions, it is necessary to design and calculate the horizontal well gravel packing length, and analyze the influence of construction parameters on the packing length, which is of great significance for reasonable design and optimization.

\section{Calculation of Equilibrium Velocity in $\alpha$ - Wave Packing Stage}

Horizontal gravel packing is essentially a solid/liquid twophase flow process that carrier fluid carrying gravel particles. When a fluid-gravel mixture is pumped through the pipe until a cross over tool where the flow will be diverted to the wellbore/screen annulus. The transport of solids in the form of a slurry has been used many years and the flow of a slurry differs from that homogeneous liquids [4]. The flow process of carrier fluid carrying gravel particles in a horiaontal wellbore can present several different flow characteristics. Generally these can be divided into following kinds: symmertric suspension, asymmetric suspension, sedimentation with a moving bed of particulates, deposited stationary bed with saltation and complete settling of the particles from the liquid. Due to its complexity, the project is generally not on the study of specific flow pattern, but uses the concept of critical or equilibrium velocity. From the initial equilibrium sand dune $[1,5-7]$ to the $\alpha$ - $\beta$ wave theory proposed by Penberthy in 1996, the $\alpha$ - $\beta$ wave packing theory has been adopted by the industry.

According to the actual packing tool combination, the carrier fluid mainly carries gravel forward in the wellbore annulus in the packing process. While part of the carrier fluid will be diverted into the washpipe/screen annulus through the screen, and the other part of the carrier fluid will leak into the formation around the wellbore through the wellbore wall. Due to the increase of wellbore annulus cross section area, carrier fluid shunt and carrier fluid leakage, the carrying capacity of carrier fluid decreases, some gravel particles will deposit and fall down to form sand dune. Under the condition of constant wellhead pump rate, the suspension and settlement of gravel particles in the wellbore annulus will reach a critical state. The sedimentary dune under this critical condition is called the critical sand bed, and the corresponding flow velocity is the equilibrium flow velocity in the $\alpha$ wave packing stage, which is an important parameter to calculate the height of the $\alpha$ wave sand bed. Chen et al. $[8,9]$ compared three equilibrium flow velocities according to the results of Gruesbeck, Penberthy and Oroskar et al.

Gruesbeck model

$$
v_{*}=15 v_{s}\left[\frac{r_{H} v_{s} \rho_{l}}{\mu_{l}}\right]^{0.39}\left[\frac{d_{p} v_{s} \rho_{l}}{\mu_{l}}\right]^{-0.73}\left[\frac{\rho_{p}-\rho_{l}}{\rho_{l}}\right]^{0.17}\left[C_{*}\right]^{0.14}
$$

Penberthy model

$$
v_{*}=\max \left(v_{1}, v_{2}\right)
$$

Where

$$
\begin{gathered}
v_{1}=\left[0.0251 g d_{p}\left(\frac{\rho_{p}-\rho_{l}}{\rho_{l}}\right)\left(\frac{D_{H} \rho_{m}}{\mu_{l}}\right)^{0.775}\right]^{0.816} \\
v_{2}=1.35\left[\frac{2 g D_{H}\left(\rho_{p}-\rho_{l}\right)}{\rho_{l}}\right]^{0.25}
\end{gathered}
$$

Oroskar and Turian model

$\frac{v_{*}}{\sqrt{g d_{p}(s-1)}}=\left\{5 C_{*}\left(1-C_{*}\right)^{2 n-1}\left(\frac{D_{H}}{d_{p}}\right)\left(\frac{D_{H} \rho_{l} \sqrt{g d_{p}(s-1)}}{\mu_{l}}\right)^{\frac{1}{8}} \frac{1}{x}\right\}^{8 / 15}$

Where $v_{*}$-critical or equilibrium velocity, $\mathrm{m} / \mathrm{s} ; v_{s}$-settling velocity of gravel particles, $\mathrm{m} / \mathrm{s} ; r_{H}$-hydraulic radius, $\mathrm{m}$; $\rho_{l} 、 \rho_{p} 、 \rho_{m^{-}}$carrier fluid density, gravel density and slurry density, respectively, $\mathrm{kg} / \mathrm{m}^{3} ; d_{p}$-gravel particle diameter, $\mathrm{m}$; $D_{H}$-hydraulic diameter, $\mathrm{m} ; \mu_{l}$-carrier fluid viscosity, Pa.s; $C_{*}$-gravel particle volume concentration under equilibrium condition, $\mathrm{m}^{3} / \mathrm{m}^{3}$; g-gravity acceleration, $\mathrm{m} / \mathrm{s}^{2} ; x$ - the correction factor for dissipation of turbulent energy, which can be written as follows:

$$
x=\frac{4}{\pi} \gamma \exp \left(\frac{-4 \gamma^{2}}{\pi}\right)+\frac{\sqrt{\pi}}{2} \operatorname{erfc}\left(\frac{2 \gamma}{\sqrt{\pi}}\right)
$$

In this relation $\gamma$ is the ratio of particle settling velocity to critical velocity. In the calculation, $x$ is determined for a range of values for $\gamma$. For the settling velocity it is observed and for a reasonable range of critical velocities $(0.018$ to $1.6185 \mathrm{~m} / \mathrm{s})$, $x \approx 0.96 ; s$-the solid/liquid density ratio, $s=\rho_{p} / \rho_{l}$. 
TABLE 1: simulation parameters.

\begin{tabular}{lccc}
\hline & \multicolumn{4}{l}{ Simulation calculation parameter list } & & \\
\hline Horizontal section length $(\mathrm{m})$ & 600 & Washpipe OD(in) & 3.8 \\
Vertical depth $(\mathrm{m})$ & 3720 & Washpipe ID(in) & 3.22 \\
Fracture gradient $(\mathrm{MPa} / 100 \mathrm{~m})$ & 1.59 & Carrier fluid leakoff ratio & $5 \%$ \\
Openhole diameter(in) & 8.75 & Design initial pump rate $(\mathrm{bpm})$ & 5.6 \\
String OD(in) & 4.11 & Sand ratio(ppg) & 0.5 \\
String ID(in) & 3.2 & Gravel diameter $(\mathrm{mm})$ & 0.33 \\
Screen OD(in) & 6.2 & Gravel volume density $\left(\mathrm{kg} / \mathrm{m}^{3}\right)$ & 1260 \\
Screen ID(in) & 4.5 & Apparent gravel density $\left(\mathrm{kg} / \mathrm{m}^{3}\right)$ & 2200 \\
Formation pressure $(\mathrm{MPa})$ & 44.2 & Carrier fluid density $\left(\mathrm{kg} / \mathrm{m}^{3}\right)$ & 1300 \\
\hline
\end{tabular}

The three equilibrium velocity correlations (1), (2) and (4) above are applicable to different situations. The equation (1) demonstrates that the equilibrium velocity depends on the particle concentrations, fluid viscosity, inertial, gravitational, and buoyant forces that act on the particles transported over the equilibrium bank. Four dimensionless groups that include forces are: the Reynolds number based on the open channel above the $\alpha$ wave bank, the Reynolds number based on the particle diameter, dimensionless density difference between the particle and the fluid, and the particle concentration. The equation (2) utilized a different equilibrium velocity model to predict transport velocity in a field-scale physical model by using gravel of the sizes $40 /$ $60,20 / 40$, and 12/20 U.S. mesh. The equation (4) developed by Oroskar and Turian, accounted for dissipation of turbulent energy. Based on the application range of three correlation formulas, parameters can be optimized and the corresponding equilibrium flow velocity can be calculated.

\section{Calculation of Flow Friction Resistance at Different Stages and $\alpha$ Wave Sand Bed Height}

3.1. The Flow Friction Resistance in $\alpha-\beta$ Wave Packing Stage. In the gravel packing process of horizontal wells, the slurry and carrier fluid flow through different locations, at different times and at different packing stages, accompanied by pressure loss and corresponding flow resistance. Especially in the gravel packing process of deepwater horizontal wells, the control of packing pressure is very important. In long horizontal wells under the environment of low formation fracture pressure, the high packing pressure will fracture formation, causing large amounts carrier fluid into the formation. In $\alpha$ wave packing stage, most of the carrier fluid flow in the wellbore/screen annulus. In $\beta$ wave packing stage, carrier fluid radial flow through the screen and into the washpipe/screen annulus axial flow and finally in the wellbore toe back into the wash pipe. As the $\beta$-wave packing stage progresses, the flow distance of carrier fluid increases gradually in the washpipe/screen annulus, so the wellbore pressure and pump pressure increase rapidly in this stage. When the $\beta$-wave reverse packing reaches the heel of the wellbore, the packing pressure reaches the maximum value.
For the specific calculation model of flow resistance in each stage, can refer to reference [10].

According to the friction model corresponding to different stages, the corresponding software is compiled to calculate the gravel packing operation parameters given for a certain horizontal well. The specific simulation parameters are shown in Table 1.

It can be seen from Figure 1 that:(1) slurry injection stage: The maximum friction resistance occurs in drill string during slurry injection stage, followed by flow friction resistance in washpipe. Since there is no sand bed formed in horizontal wellbore annulus during slurry injection stage, only pure fluid flows, so the horizontal wellbore friction resistance is relatively small. (2) the $\alpha$ packing stage: due to the long drill string length in deepwater formation, the friction resistance in the drill string is still the biggest, but the proportion is slightly lower. The friction resistance in the wash pipe stays the same, but the proportion has decreased. As the formed sand bed move forward gradually in the $\alpha$ packing stage, the friction resistance in the upper sand bed increases gradually, and carrier fluid friction resistance at sand bed front gradually reduce; (3) the $\beta$ wave packing stage: in the reverse packing process, flow length for carrier fluid in washpipe/screen annulus increases gradually, friction resistence increases rapidly. Meanwhile, for the sand bed covered screen, in the process of the reverse packing, flow length decreases, and the seepage velocity through sand bed increases, the flow friction will increase. While the friction resistance proportion for slurry injection in the drill string decreases.

Overall, pressure loss occurs mainly in the $\beta$ reverse packing stage, especially when the $\beta$ wave approaches the heel of the horizontal well. The carrier fluid flows through the long distance and the narrow washpipe/screen annulus and enters washpipe at the inlet of wellbore toe. A rapid increase in friction resistance leads a rapid increase in packing pressure. It is easy to break through the formation fracture pressure. Once the formation fracture pressure is broken through, a large amount of carrier fluid will leak into the formation, resulting in packing failure.

3.2. Calculation of $\alpha$ Wave Sand Bed Height. The design of $\alpha$ wave sand bed height is a key step in the gravel packing of the entire horizontal well $[11,12]$. In this process, premature 
Friction resistance distribution for gravel pack

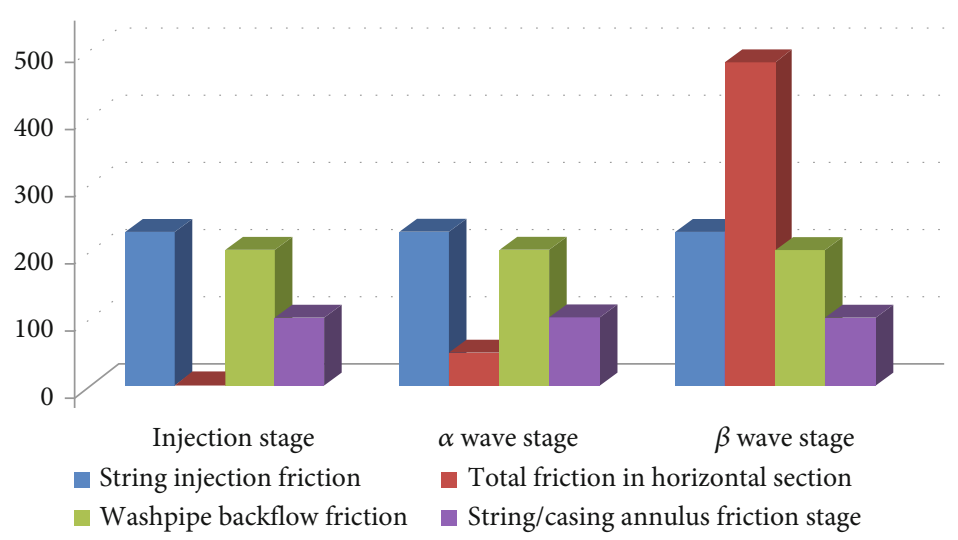

FIgURE 1: Comparison and analysis of 3-stage friction distribution of gravel packing in horizontal Wells.

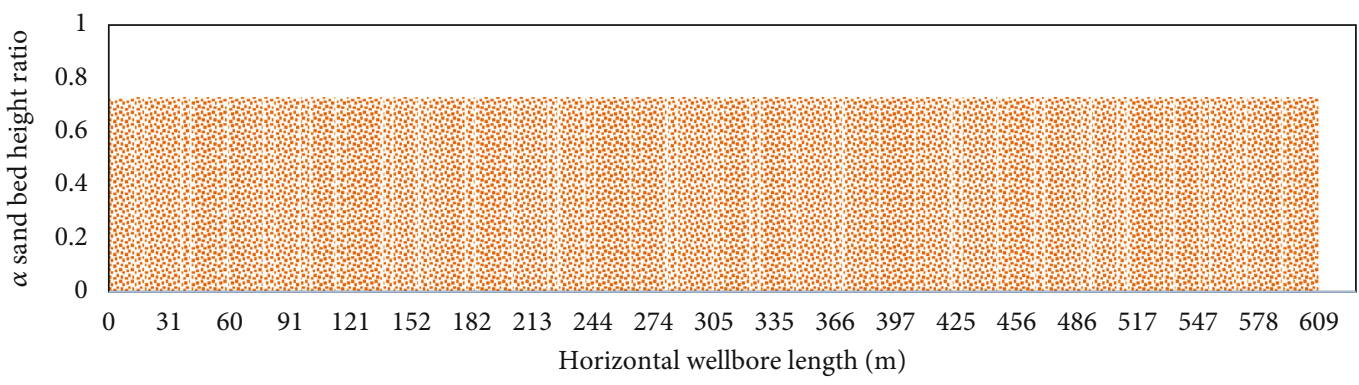

FIgURE 2: Calculation result for $\alpha$ wave sand bed height.

sand bridge is prone to be formed if the designed sand bed is too high. The gravel concentration in wellbore/screen annulus will increase with the carrier fluid leakage along packing process, resulting in high sand bed height. According to the critical sand bed theory of $\alpha$ - $\beta$ wave packing, the calculation method and calculation process of $\alpha$ wave sand bed height are realized. The specific calculation process is as follows:

(1) Given the size parameters of the well structure, washpipe and screen system;

(2) Select carrier fluid (density and viscosity), gravel parameters and specific pump rate $Q$;

(3) The horizontal section was separated into $N$ sections and the packing calculation was carried out step by step;

(4)For packing section $i_{t h}$, according to initial pump rate $Q$ 、 the amount of leakage rate calculated before $(i-1)_{t h}, q_{p}$ and the former $(i-1)$ section enters the washpipe/screen annulus return flow rate $q_{r}$, Calculate the actual flow rate of section $i_{t h}, Q_{i}=Q-q_{p}-q_{r}$;

(5)According to the actual flow $Q_{i}$ of section $i_{t h}$ and the current sand bed height $h$, the wellbore annular crosssectional area $S$ was calculated, and the velocity $v$ of sand bed upper was obtained;

(6) According to the equilibrium of velocity model in (1), determine the actual velocity and the equilibrium flow velocity, whether to meet $|v-v *|<\varepsilon$, if yes, then the sand bed height $h=h+d h$, if not, return to step (5) recalculate until the accuracy of critical velocity is satisfied, and record as the equilibrium sand bed height at section $i_{t h}$;
(7) Step by step as above (4)-(6), until the packing reaches the $N_{t h}$ stage, complete.

Using the basic data in Table 1 and according to the above calculation process, the corresponding software was compiled to calculate the sand bed height, as shown in Figure 2.

As can be seen from Figure 2, if there is no loss of carrier fluid, the $\alpha$ wave sand bed height along wellbore is in a straight line; if the carrier fluid leakoff ratio along the wellbore into the around reservoir is low $(5 \%)$, the the $\alpha$ wave sand bed height is basically a constant along the wellbore. In this case, as long as the the $\alpha$ wave sand bed height is designed within a safe range, the $\alpha$ wave stage can be completed. If the carrier fluid leakoff is high, the amount of carrier fluid in the wellbore annulus along the wellbore will be reduced. The carrying capacity of carrier fluid will be reduced, resulting in premature settlement of excess gravels, which makes the the $\alpha$ wave sand bed height increase rapidly, and it is easy to cause premature sand bridge and packing failure.

\section{Determination Method of Packing Length in $\alpha$ Wave Packing Stage}

Premature bridge can occur if pump rate is too low and carrier fluid is not sufficient to support gravel to the toe of the wellbore in $\alpha$ wave packing stage. If there is a leakoff of carrier fluid along the path, the slurry flow rate will be reduced, and the carrying capacity of carrier fluid will be reduced. To 


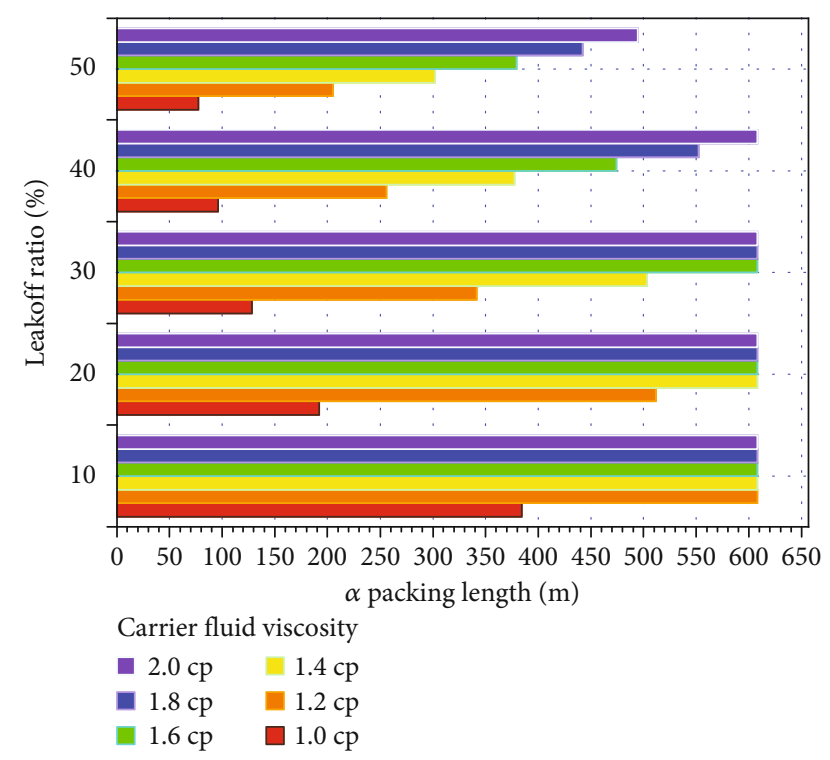

FIGURE 3: Effect of viscosity and leakoff on $\alpha$ wave packing length.

be able to carry gravel to the toe of the wellbore, there needs sufficient flow rate and energy to guide the carrier fluid through the toe inlet into the washpipe and then return along the washpipe. Experimental studies and field operation data show that [2], for the determined gravel concentration, the apparent velocity of carrier fluid at the toe of wellbore should not be less than $1 \mathrm{ft} / \mathrm{s}$ [2]. If the apparent velocity is lower than this, the $\alpha$ wave packing process will stop and reverse packing will begin. At this time, an effective means is to reduce the sand ratio appropriately [13].

According to different string structure and packing parameters, the upper limit of sand dune ratio (sand bed height/wellbore diameter) can be set as 0.8 . If the sand dune ratio exceeds this value during the $\alpha$ wave packing process, it is considered that a sand bridge is formed, resulting in early plugging and the $\alpha$ wave packing process is ended. The $\beta$ wave reverse packing starts at plugging location and the length from the heel of wellbore to plugging location is $\alpha$ wave packing length. There are many factors influencing the length of $\alpha$ wave packing [12-16], including pump rate, sand ratio, carrier fluid leakoff, washpipe/screen ratio, carrier fluid viscosity, fluid density and gravel density. For gravel packing of long horizontal wells in deepwater, the successful completion of $\alpha$ wave packing is an important first step.

Using the basic data in Table 1, designed the upper limit of sand dune ratio is 0.8 , carrier fluid leakoff ratio separately: $10 \%, 20 \%, 30 \%, 40 \%, 50 \%$, carrier fluid viscosity were taken: $1.0,1.2,1.4,1.6,1.8,2.0 \mathrm{cp}$, respectively, to calculate the effect of leakoff rate, carrier density and viscosity on the length of $\alpha$ wave packing.

Figure 3 shows the change of $\alpha$ wave packing length under different combinations of carrier fluid viscosity and carrier fluid leakoff ratio. For low viscosity carrier fluid, gravel transport mainly depends on the flow rate of carrier fluid to provide carrying capacity. If the leakoff rate is higher, the flow rate in annulus will decrease and more gravel will settle. The sand dune ratio will be higher than designed value in advance and premature sand bridge will occur. At this time, the $\alpha$ wave packing length is shorter. With the increase of the viscosity of the carried, the suspension capacity of carrier fluid in increased and keeps a certain amount of gravel in suspension.

Although the leakoff rate of the carrier fluid increases, the gravel can still be carried forward by the viscosity of the carrier fluid. With the increase of the viscosity of the carrier fluid, the corresponding $\alpha$ wave packing length is longer. The larger the leakoff rate, the shorter the $\alpha$ wave packing length under the same the viscosity of the carrier fluid.

Figure 4 shows the influences of different carrier fluid densities and leakoff ratio on the $\alpha$ wave packing length. Conventional gravel was used at this time, and the carrier fluid densities were, respectively, 1.250, 1.275, 1.300, 1.325 and 1.350sg, the $\alpha$ packing length increases with the increase of the carrier fluid density, because the density difference between the carrier fluid and gravel particles decreases. When the leakoff ratio is greater than $20 \%$, the corresponding carrier fluid density cannot achieve the desired wellbore length.

\section{Determination Method of Complete Packing Length of $\alpha-\beta$ Wave Packing}

Under certain parameters, ideal gravel pack requirement is that the $\alpha$ wave packing and $\beta$ wave reverse packing can be successfully completed. As the previous 3 , complete $\alpha$ packing must meet $\alpha$ sand dune ratio should not exceed the value of design and sufficient return rate at the inlet of washpipe $[17,18]$. Throughout the $\beta$-wave reverse packing stage, the packing pressure increases dramatically due to the rapid increase friction in washpipe/screen annulus. Once the packing pressure exceeds the formation fracture pressure, a large amount of carrier fluid is lost. The distance from the pressure breakthrough point to the toe of the wellbore is called the $\beta$-wave reverse packing length. If $\alpha$ packing length is equal to the horizontal section length from the heel to the toe of the horizontal wellbore, while the $\beta$ wave reverse packing length is equal to the horizontal section length from the toe to the heel of the horizontal wellbore, known as $\alpha-\beta$ wave complete packing length. Whether early sand bridge in $\alpha$ wave packing stage, or pressure breakthrough of formation fracture pressure in $\beta$ wave packing stage, all is not completed successfully $\alpha-\beta$ packing. In general, the packing length is the length of $\alpha-\beta$ wave successfully completed. The principle of designing the packing length is: the dune ratio in the process of $\alpha$ wave packing is lower than 0.8 , and the packing pressure in the process of $\beta$ wave packing is less than the formation fracture pressure. According to this design principle, the corresponding software is compiled, and the calculation is carried out by using the basic data in Table 1, and some factors affecting the packing length are analyzed.

For conventional gravel (2.2sg gravel density), the leakoff ratio was set to $5 \%$ with three different densities of carrier fluids: $1.25 \mathrm{sg}, 1.30 \mathrm{sg}$, and $1.35 \mathrm{sg}$. Figure 5 shows the corresponding simulation results. 


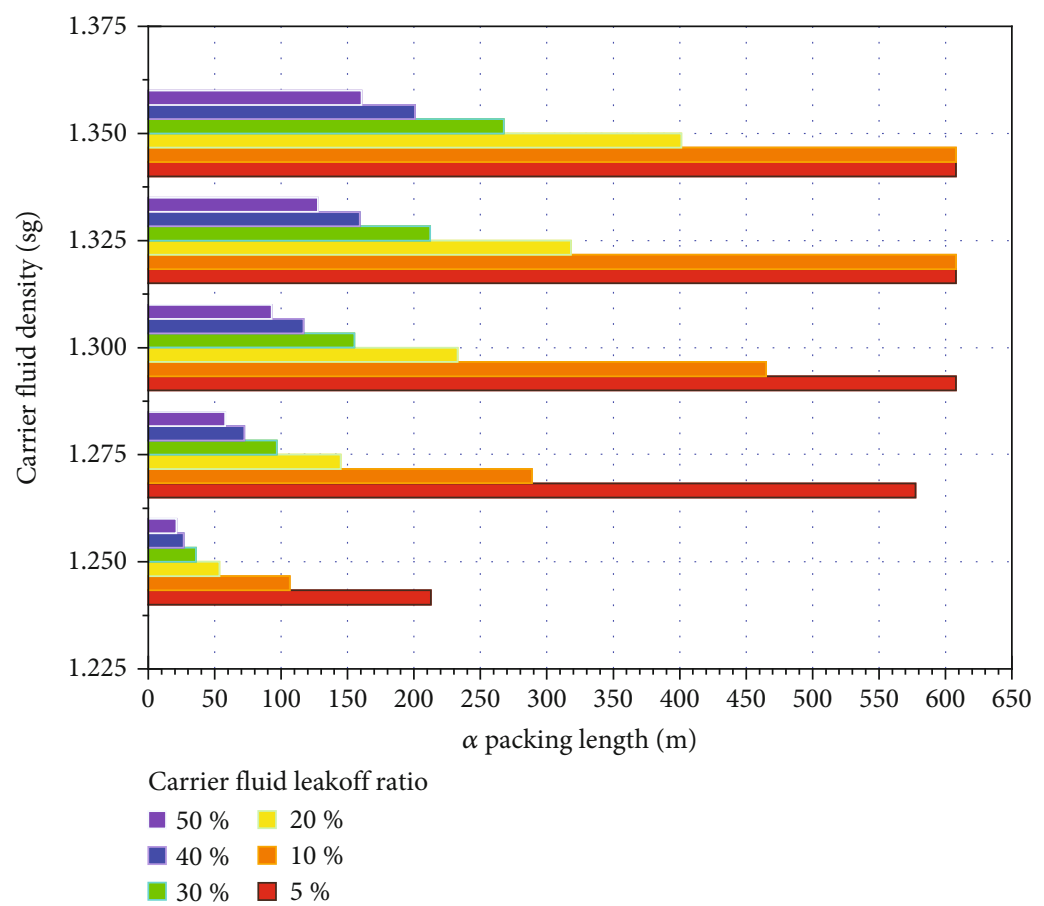

FIGURE 4: Influence of density and leakoff of carrier fluid on $\alpha$ packing length.

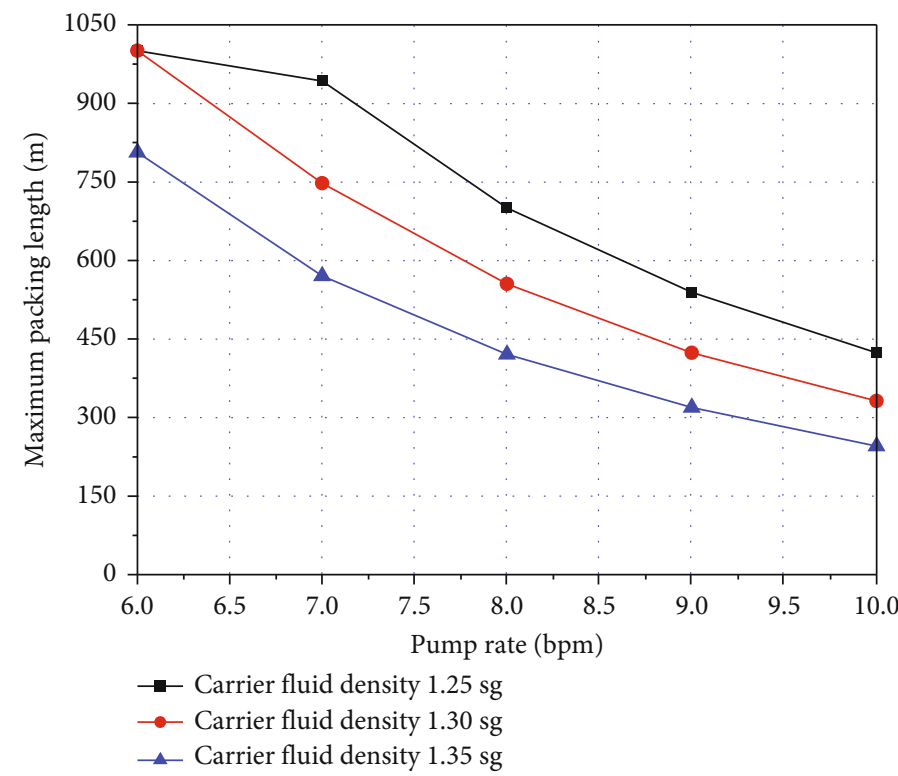

FIGURE 5: Influence of pump rate and fluid density on maximum packing length.

It can be seen from Figure 5 that, with the increase of pump rate, the packing lengths corresponding to the densities of the three carrier fluid gradually decrease. Although it is helpful for $\alpha$ wave packing because of the increase of pump rate, packing pressure increases rapidly in the $\beta$ wave packing stage, and it is easy to break through the formation rupture pressure. If the pump rate is lower than $6.0 \mathrm{bpm}$, sand bridge will be formed early in the $\alpha$ wave packing stage, and effective $\alpha$ wave packing length cannot be reached. Under the condition of a certain pump rate, the complete $\alpha-\beta$ wave packing length decreases with the increase of the density of carrier fluid. This is because the high density of carrier fluid in the $\beta$ wave packing stage leads to high friction in washpipe/screen annulus, resulting in rapid growth of packing pressure, which leads to early pressure breakthrough and reduces the $\beta$ wave reverse packing length. The density of carrier fluid was 1.3sg, and Figure 6 simulated the effect of four different gravel densities on the pack length.

It can be seen from Figure 6 that for higher pump rate, the $\alpha$-wave sand bed height can be controlled to successfully complete the $\alpha$-wave packing stage. However, for $\beta$-wave 


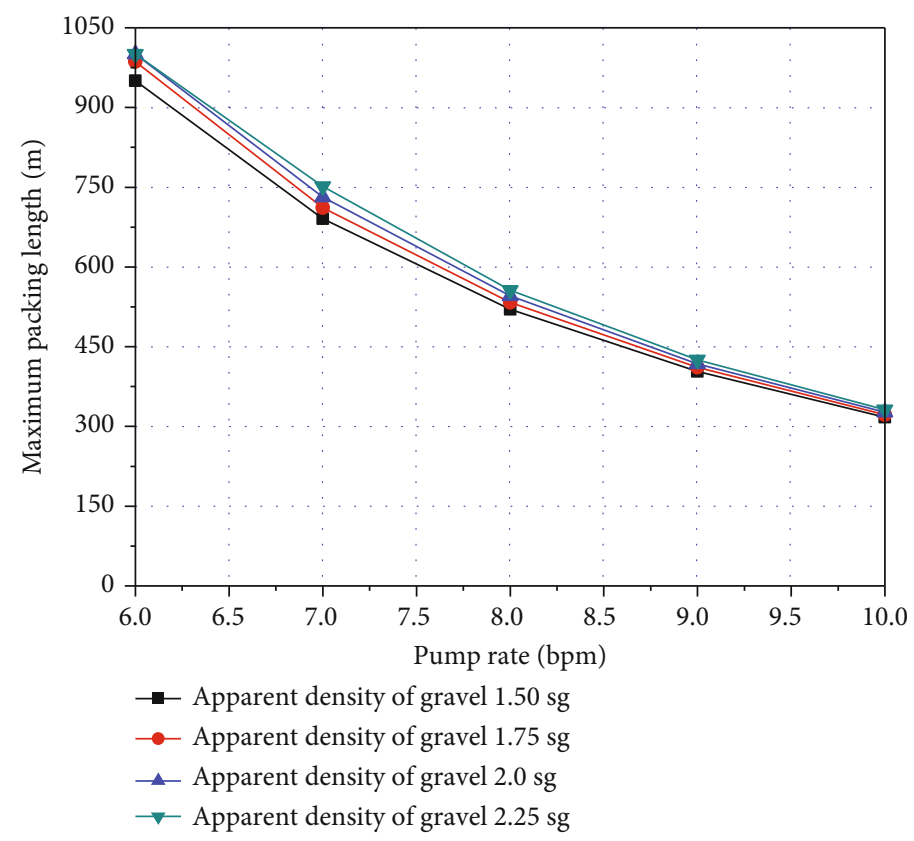

FIGURE 6: Effect of different gravel density on packing length.

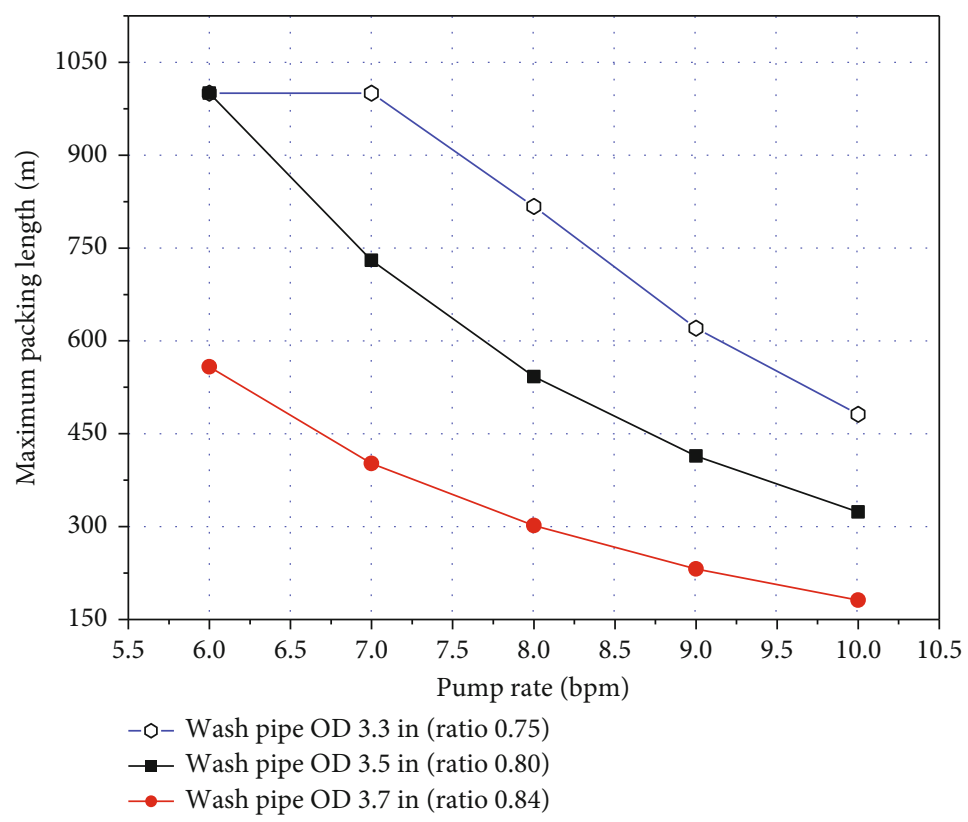

FIGURE 7: Change of packing length with pump rate under washpipe/screen (screen ID: 4.4in).

packing stage, the reverse packing length of $\beta$-wave is mainly affected by the packing pressure $[19,20]$. With the increase of pump rate, the complete $\alpha-\beta$ wave packing length decreases.

The influence of screen and washpipe size on gravel pack length is discussed below. Screen ID is 4.4in, washpipe OD is 3.3in,3.5in and 3.7in, respectively, and basic calculation parameters are shown in Table 1. According to the complete $\alpha$ - $\beta$ wave packing length method designed above, the change of packing length with pump rate is calculated under three different washpipe/screen ratios(washpipe OD/screen ID).
Three cases of washpipe/screen ratio were 0.750 .80 and 0.84 . If the screen size does not change, the greater washpipe/screen ratio is (that is, the greater the wash pipe diameter), the smaller the clearance of washpipe/screen annulus is. Flow friction resistance in the washpipe/screen annulus will increase and can ensure more carrier fluid in the wellbore/screen annulus. The carrying capacity of carrier fluid increases. Increased carrying capacity in the wellbore annulus helps with $\alpha$ wave packing. During $\beta$-wave packing, especially late in the $\beta$-wave packing process, because the carrier fluid has to flow through the narrow washpipe/screen 
annulus, which is longer in long horizontal wellbore, the high pressure loss will be caused. This increases the $\beta$-wave packing pressure, which can easily exceed the formation fracture. On the contrary, if the washpipe/screen ratio is small, the washpipe/screen annulus becomes larger. At this time, more carrier fluid will be diverted to the washpipe/ screen annulus, and then enters the washpipe at the toe entrance. Due to more carrier fluid diversion, the carrying capacity of carrier fluid in the wellbore/screen annulus is reduced, and early sand blocking is easy to occur. Can be seen from the Figure 7, with the increase of the washpipe/ screen ratio, $\alpha-\beta$ conplete packing length decreases. This is because the packing pressure increases rapidly in the $\beta$ -wave stage after the increase of the washpipe/screen ratio, resulting in the shortening of the length of the $\beta$-wave reverse packing and the decrease of the length of the complete $\alpha$ - $\beta$ wave packing. For specific horizontal gravel pack, parameters such as washpipe/screen ratio need to be optimized. According to experiment and field experience, the washpipe/screen ratio is generally about 0.8 .

\section{Conclusions}

(1) According to the mechanism of gravel packing in horizontal wells, precise calculation and analysis of friction resistance in different stages are carried out. In the entire packing stage, the packing pressure increases rapidly in the $\beta$ wave packing stage and it is easy to break through the formation fracture pressure

(2) Based on the equilibrium flow velocity calculation model, the calculation method and calculation process of $\alpha$ wave sand bed height are presented

(3) The characteristics of $\alpha$-wave sand bed is studied, especially in the gravel packing of long horizontal well in deepwater. The conditions of forming stable $\alpha$-wave equilibrium sand bed are studied. The calculation model and process of $\alpha$-wave packing length are established, and the quantitative variation law of parameters affecting $\alpha$-wave packing length is discussed

(4) The design principle and method of complete $\alpha-\beta$ wave packing length are put forward, and the influencing of parameters such as carrier fluid density, carrier fluid viscosity, pump rate and washpipe/screen ratio on complete $\alpha-\beta$ wave packing length are analyzed by using the software

(5) By using the established model and method, gravel packing parameters can be optimized to provide support for the success of field gravel packing construction

\section{Data Availability}

This is a theoretical work for which no data need to be made available.

\section{Conflicts of Interest}

The authors declare that they have no conflicts of interest.

\section{Acknowledgments}

This work was supported by the National Natural Science Foundation of China Project "Basic Research on Wellbore Pressure Control in Deep Water Oil and Gas Drilling and Production (no. 51734010)".

\section{References}

[1] C. Gruesbeck, W. M. Salathiel, and E. E. Echols, "Design of gravel packs in deviated wellbores," Journal of Petroleum Technology, vol. 31, no. 1, pp. 109-115, 1979.

[2] W. L. Penberthy, K. L. Bickham, H. T. Nguyen, and T. A. Paulley, "Gravel placement in horizontal wells," in SPE Formation Damage Control Symposium, Lafayette, LA, 1996.

[3] Z. Chen and R. Novotny, "The dynamic bottomhole pressure management: A necessity to gravel packing long horizontal wells with low fracture gradients," in Paper SPE 96396, presented at the 2005 SPE Annual Technical Conference, pp. 912, Dallas, Texas, U.S.A., October 2005.

[4] G. W. Govier and K. Aziz, The Flow of Comples Mixtures in Pipes, Robert E. Krieger Publishing Co. Inc., Huntington, NY, 1972.

[5] A. R. Oroskar and R. M. Turian, "The critical velocity in pipeline flow of slurries," AICHE Journal, vol. 26, no. 4, pp. 550558,1980

[6] J. X. Forrest, "Horizontal gravel packing studies in a full-scale model wellbore," in Paper SPE 20681, presented at the 1990 SPE Annual Technical Conference, pp. 23-26, New Orleans, LA, September, 1990.

[7] S. D. E. Jr, "Gravel-pack studies in a full-scale, high-pressure wellbore model," in Paper SPE 16890, presented at the 1987 SPE Annual Technical Conference, pp. 27-30, Dallas, 1987.

[8] Z. Chen, "Horizontal well gravel packing: Dynamic alpha wave dune height calculation and its impact on gravel placement job execution," in Paper SPE 110665, presented at the 2007 SPE Annual Technical Conference, pp. 11-14, Anaheim, California, U.S.A., November 2007.

[9] Z. Chen, R. J. Novotny, R. Farias, A. Mendez, C. A. Pedroso, and L. H. Fernandes, "Gravel packing deep water long horizontal wells under low fracture gradient," in Paper SPE 90552, presented at the SPE Annual Technical Coference, pp. 26-29, Houston, Texas, September 2004.

[10] A. L. Martins, J. V. M. de Magalhaes, A. Calderon, and C. M. Chagas, "A mechanistic model for horizontal gravel pack displacement," in Paper SPE 82247, presented at the SPE European Formation Damage Conference, pp. 13-14, The Hague, The Netherlands, May 2003.

[11] L. Aifen and Y. Liu, "Numerical simulation study of gravel packing in horizontal well," Acta Petrol EI Sinica, vol. 23, no. 1, pp. 102-107, 2002.

[12] Z. Shengtian, L. Zhaomin, and D. Changyin, "The advances of numerical simulation for gravel packing in horizontal wells," Mechanics in Engineering, vol. 31, no. 3, pp. 9-15, 2009.

[13] J. Jeanpert, T. Banning, C. Abad, I. Mbamalu, and J. Hardy, "Successful installation of horizontal openhole gravel-pack completions in low fracture gradient environment: A case 
history from deepwater West Africa," in Paper SPE-189492MS, presented at the SPE International Conference and Exhibition on Formation Damage Control, Lafayette, Louisiana, U.S.A., 2018.

[14] D. J. Attong and W. V. C. DeLandro, "Case History: Pushing The Limit On Long Horizontal Gravel Packs," in Paper SPE 36087, presented at the Fourth Latin American and Caribbean Petroleum Engineering Conference, pp. 23-26, Port of Spain, Trinidad, April 1996.

[15] K. Alexander, D. Bruce, C. Williamson et al., "Evolution of Open-Hole Gravel Pack Methodology in a Low FracWindow Environment: Case Histories and Lessons Learned From the Kraken Field Development," in Paper SPE-195937MS, presented at the SPE Annual Technical Conference, Calgary, Alberta, Canada, October 2019.

[16] T. Hubert, M. T. Chembou, N. Dupouy, R. Ilyasov, C. M. Nkala, and B. Reilly, "Optimised pumping design for $100 \%$ pack efficiency in open-hole gravel packs: A field study from Offshore Republic of Congo," in Paper SPE-202291-MS, presented at the SPE Asia Pacific Oil \& Gas Conference, Perth, Australia, October 2020.

[17] J. A. Firth, H. Hanid, and P. M. Saldungary, "Novel techniques enable successful gravel packing of long horizontal North Sea wells," in SPE Europec, Amsterdam, 2020.

[18] V. Gupta, J. Jeanpert, C. John et al., "Analysing critical elements in openhole gravel pack treatment design cycle: Case study in Indian ultra-deepwater," in SPE Asia Pacific Oil \& Gas conference and Exhibition, Perth, Australia, November 2020.

[19] F. C. Colbert, A. L. Costa, R. F. Gachet, F. M. Garcia, and A. J. Neto, "Sand control technology for extend-reach wells: Best practices from the longest gravel packs performed Offshore Brazil," in International Petroleum Exhibition \& Conference, Abu Dhabi, UAE, November 2017.

[20] M. P. Coronado and T. G. Corbett, "Beta-wave pressure control enables extended-reach horizontal gravel packs," in 2001 SPE annual technical conference and exhibition, New Orleans, Louisiana, October 2001. 